\title{
Why Market Orientation Matters for Agriculture and Fishery Workers? Unravelling the Association between Occupational Background and Caloric Deprivation in India
}

Smita Kumar ( $\square$ smita.greenearth@gmail.com )

Sun Yat-Sen University https://orcid.org/0000-0003-4081-6154

Sunil Rajpal

Institute of Economic Growth

Shiau-Yun Lu

Sun Yat-Sen University

William Joe

Institute of Economic Growth

Research article

Keywords: Household Consumer Expenditure Survey (HCES), National Sample Survey (NSS)

Posted Date: August 12th, 2020

DOI: https://doi.org/10.21203/rs.3.rs-50100/v1

License: @ (i) This work is licensed under a Creative Commons Attribution 4.0 International License.

Read Full License

Version of Record: A version of this preprint was published at BMC Public Health on April 8th, 2021. See the published version at https://doi.org/10.1186/s12889-021-10644-9. 


\section{Abstract}

Background: Given the presumptions of low-productivity, developmental policies in low- and middleincome countries usually display a pro-manufacturing bias and largely undermine the potentials within the agriculture sectors in promoting sustainable growth and development. As a consequence, skill development is usually aligned with preferences of the manufacturing-led economic growth and is accorded high priorities in policymaking. However, the success of such skill development policies essentially hinges upon the assumption of a quick and successful structural transformation towards nonagricultural sector (manufacturing and services). But contrary to expectations, the pace of economic transition has been rather slow in India and almost one-half of the workforces continue to be engaged in agriculture and allied activities. Moreover, it is unclear whether such shift away from agriculture can necessarily lead to reductions in widespread deprivations in the form of food insecurity and under nutrition. In fact, it is also feasible that market orientation of skilled agricultural and fishery sector may display favorable impact on poverty and nutritional well-being of households.

Methods: Therefore, drawing upon data from $68^{\text {th }}$ round (2011-12) of nationally representative crosssectional Household Consumer Expenditure Survey (HCES) of National Sample Survey (NSS), Government of India, this study aims to examine the association between occupational backgrounds and nutritional deprivation (average caloric consumption as well as low calorie intake) among Indian households.

Results: Evidences show that agricultural and fishery labor households have lowest calorie intake (2086 kcal) across all the occupational groups. However, market oriented skilled agricultural and fishery workers' (2261 kcal - rural, $2165 \mathrm{kcal}$ - urban) have higher calorie intakes than those belonging to subsistence agricultural ( $2165 \mathrm{kcal}$ - rural, $2149 \mathrm{kcal}$ - urban) and fishery workers or agricultural and fishery laborer (2086 kcal - rural, $2071 \mathrm{kcal}$ - urban). Further, the multilevel logistic regression estimates suggest that in rural areas, households engaged in skilled agricultural and fishery works have significantly (at $5 \%$ level) lower odds ratio (OR: 0.72 , with $95 \% \mathrm{Cl}: 0.63 ; 0.82)$ of having insufficient calorie intake compared to the unskilled agricultural and fishery laborer households.

Conclusion: These insights when combined with the occupation-specific random-effects suggests that policy focus to promote market oriented skilled agricultural and fishery workers can be an equally good option as direct investment in professional and managerial skills for manufacturing and services.

\section{Introduction}

Given the presumptions of low-productivity, developmental policies in low- and middle-income countries usually display a pro-manufacturing bias and largely undermine the potentials within the agriculture and fishery sectors in promoting sustainable growth and development [1-5]. As a consequence, skill development is usually aligned with preferences of the manufacturing-led economic growth and is accorded high priorities in policymaking $[6,7]$. For instance, in India, the thrust on such approach is 
evident from the creation of a separate ministry for skill development and entrepreneurship (MSDE) that is concerned with policies to impart employable skills to the working-age (15-59 years) population that accounts for about two-third of the total population. It is expected that skill development can enhance employability quotient of the labor force and thus facilitate rapid reductions in poverty and deprivation. However, the success of such skill development policies essentially hinges upon the assumption of a quick and successful structural transformation towards non-agricultural sector (manufacturing and services). But contrary to expectations, the pace of economic transition has been rather slow in India and almost one-half of the workforces continue to be engaged in agriculture and allied activities [8]. Moreover, it is unclear whether such shift away from agriculture can necessarily lead to reductions in widespread deprivations in the form of food insecurity and under nutrition. For instance, despite rapid economic growth in recent years, India is poorly ranked 97th of 118 countries in the Global Hunger Index 2016 [9]. In fact, sustained declines in nutritional intake (calories as well as other nutrients) are identified as a major developmental and food security concern [10-12].

Given the context, it is critical to unravel the association between occupational background and nutritional deprivations and thereby develop insights regarding the scope and focus of skill development policies. For instance, it is noted that poverty and nutritional deprivations are generally concentrated among households belonging to unskilled occupational categories such as agricultural labor, casual labor or fishery workers [13-18]. But it is unclear whether only selected sections or the entire agricultural and fishery sector is vulnerable to such risks. Also, there is no evidence to understand the relative advantages of skilled agricultural and fishery sector vis-à-vis other occupations. In fact, it is also feasible that market orientation of skilled agricultural and fishery sector may display favorable impact on poverty and nutritional well-being of households [19-24]. Given such possibilities, this paper aims to examine the association between occupational backgrounds with nutritional deprivation (average caloric consumption as well as low calorie intake) among Indian households. As such, a focus on caloric intake is critical because it is a fundamental indicator of food security and has a major influence on economic well-being $[25,11]$. Moreover, adequate calorie intake has high relevance for nutritional well-being and is regarded as a fundamental marker of public health $[10,26]$. Given such relevance, an analysis of average per capita calorie intake can highlight the patterns of nutritional deprivation across various occupational groups and can effectively outline the differences and similarities therein. Thus, these results can also provide vital insights to decide upon policy approach towards skill development and diversification. In particular, this can help to comprehend whether promotion of market oriented skills among the unskilled agricultural and fishery households can have significant influence on nutritional and food security. The relevance of these findings increases manifold because of its wider implications for other developing countries. In fact, similar patterns of income and nutritional deprivation are observed across other lowand middle-income countries and reiterate the need and relevance of a comparative analysis of nutritional well-being across various occupational groups [27, 15, 3, 28-29].

\section{Data And Methods}


This study is based on data from nationally representative cross-sectional Household Consumer Expenditure Survey (HCES) conducted in 2011-12 (68th round) by National Sample Survey Office (NSSO), Ministry of Statistics and Programme Implementation (MOSPI), Government of India (NSSO 2014). The HCES is widely used by developmental practitioners and policymakers to assess the levels and patterns of food consumption across various population subgroups in India. The HCES uses a stratified, multistage cluster design at state-level to provide reliable estimates at state and for rural and urban areas. Households for survey are selected on the basis of circular systematic sampling. The results are estimated using data from HCES schedule 1.0 (Type 1/ Mixed Reference Period) which altogether has a sample of 1, 01,662 households (59695 rural +41967 urban).

The HCES provides occupational categories of households based on the code structure provided in the National Classification of Occupations, 2004 [30]. Based on this coding structure, the households are categorized into 27 mutually exclusive occupational categories (Table 1). Based on a mixed recall period of 30 days and 365 days, the HCES provides direct information on household food and non-food consumption. In this paper, the information on food consumption is used to estimate the average per capita per day per caloric intake (in kilocalories, kcal) across households. The estimation is based on intake conversion parameter prescribed by the NSSO and which is derived from a nutrition chart that provides details regarding energy content of different foods in the Indian diet [31]. For certain food items the intake quantity is unavailable but has been replaced by information on average energy contents per Indian rupee. It is worth noting that the consumption details are available at the household level and thus cannot be specifically associated with caloric intake of each household member. Notwithstanding this limitation, the household per capita per day calorie intake has been an important indicator to examine levels, trends and patterns in nutritional intake in India. Further, we define prevalence of under nutrition or low-calorie intake as the situation when households are estimated to be consuming less than $80 \%$ of the prescribed calorie norms ( $2400 \mathrm{kcal}$ in rural areas and $2100 \mathrm{kcal}$ in urban areas). In other words, households with per capita per day consumption of less than $1920 \mathrm{kcal}$ in rural areas and less than $1680 \mathrm{kcal}$ in urban areas are regarded as undernourished household. Use of this 80 percent threshold is motivated by the approach adopted NSSO in its analysis of levels and patterns in nutritional intake in India diet [31]. In fact, the estimates of under nutrition obtained using this benchmark is more or less similar to the proportion of nutritional deprivation estimated using other anthropometric measures such as prevalence of low body mass index among men and women in India [32]. Based on this transformation of caloric information we arrive at two outcome indicators for the analysis: average per capita per day caloric intake across households (continuous outcome) and prevalence of low-caloric intake or under nutrition across households (binary outcome). 
Table 1

Description of sample population of households in India by occupational classification, National Sample Survey, 2011-2012.

\begin{tabular}{|c|c|c|c|c|c|c|}
\hline \multirow[t]{2}{*}{ Household occupational groups (NCO) } & \multicolumn{2}{|l|}{ Rural } & \multicolumn{2}{|l|}{ Urban } & \multicolumn{2}{|l|}{ All } \\
\hline & $\mathbf{N}$ & $\%$ & $\mathbf{N}$ & $\%$ & $\mathbf{N}$ & $\%$ \\
\hline Legislators \& senior officials & 132 & 0.2 & 217 & 0.6 & 349 & 0.4 \\
\hline Corporate managers & 4,144 & 7.3 & 5,538 & 14.7 & 9,682 & 10.3 \\
\hline General managers & 40 & 0.1 & 122 & 0.3 & 162 & 0.2 \\
\hline Science professionals & 137 & 0.2 & 708 & 1.9 & 845 & 0.9 \\
\hline Life science \& health professionals & 310 & 0.5 & 371 & 1.0 & 681 & 0.7 \\
\hline Teaching professionals & 1,116 & 2.0 & 1,088 & 2.9 & 2,204 & 2.3 \\
\hline Other professionals & 1,142 & 2.0 & 1,536 & 4.1 & 2,678 & 2.8 \\
\hline Science associate professionals & 139 & 0.2 & 402 & 1.1 & 541 & 0.6 \\
\hline Life science \& health associate professionals & 255 & 0.5 & 351 & 0.9 & 606 & 0.6 \\
\hline Teaching associate professionals & 2,051 & 3.6 & 972 & 2.6 & 3,023 & 3.2 \\
\hline Other associate professionals & 787 & 1.4 & 1,433 & 3.8 & 2,220 & 2.4 \\
\hline Office clerks & 1,076 & 1.9 & 1,746 & 4.6 & 2,822 & 3.0 \\
\hline Customer services clerks & 133 & 0.2 & 299 & 0.8 & 432 & 0.5 \\
\hline Personal \& protective service workers & 2,044 & 3.6 & 2,330 & 6.2 & 4,374 & 4.6 \\
\hline Models, sales persons \& demonstrators & 3,655 & 6.5 & 3,906 & 10.4 & 7,561 & 8.0 \\
\hline $\begin{array}{l}\text { Market oriented skilled agricultural \& fishery } \\
\text { workers }\end{array}$ & 15,971 & 28.2 & 1,854 & 4.9 & 17,825 & 18.9 \\
\hline Subsistence agricultural \& fishery workers & 571 & 1.0 & 75 & 0.2 & 646 & 0.7 \\
\hline Extraction \& building trades workers & 4,566 & 8.1 & 2,608 & 6.9 & 7,174 & 7.6 \\
\hline Metal, machinery \& related trades workers & 910 & 1.6 & 1,379 & 3.7 & 2,289 & 2.4 \\
\hline $\begin{array}{l}\text { Precision, handicraft, printing \& related trades } \\
\text { workers }\end{array}$ & 358 & 0.6 & 423 & 1.1 & 781 & 0.8 \\
\hline Other craft \& related trades workers & 1,666 & 2.9 & 1,604 & 4.3 & 3,270 & 3.5 \\
\hline Stationary plant \& related operators & 295 & 0.5 & 332 & 0.9 & 627 & 0.7 \\
\hline Machine operators \& assemblers & 633 & 1.1 & 931 & 2.5 & 1,564 & 1.7 \\
\hline
\end{tabular}

Source: Data from National Sample Survey, Government of India 


\begin{tabular}{|lllllll|}
\hline Household occupational groups (NCO) & \multicolumn{2}{l}{ Rural } & \multicolumn{3}{c}{ Urban } & \multicolumn{3}{c|}{ All } \\
\cline { 2 - 8 } & $\mathbf{N}$ & $\%$ & $\mathbf{N}$ & $\%$ & $\mathbf{N}$ & $\%$ \\
\hline Drivers \& mobile-plant operators & 2,137 & 3.8 & 1,950 & 5.2 & 4,087 & 4.3 \\
\hline Sales \& services elementary occupations & 1,235 & 2.2 & 1,904 & 5.1 & 3,139 & 3.3 \\
\hline Agricultural, fishery \& related labourers & 5,571 & 9.9 & 820 & 2.2 & 6,391 & 6.8 \\
\hline $\begin{array}{l}\text { Mining, construction, manufacturing \& } \\
\text { transport labourers }\end{array}$ & 5,462 & 9.7 & 2,722 & 7.2 & 8,184 & 8.7 \\
\hline All households & 56,536 & 100 & 37,621 & 100 & 94,157 & 100 \\
\hline Source: Data from National Sample Survey, Government of India & & & & \\
\hline
\end{tabular}

The analysis is conducted separately for rural and urban areas as they have different average calorie intake norms. It may be noted that the analysis is based on a sample of 94,157 households (56536 rural, 37621 - urban) after filtering out observations where household NCO codes or other correlates are missing or not specified. Following a bivariate analysis, we use multilevel linear and logistic regression models to understand the association of occupational groups with continuous and binary outcomes, respectively. The analysis is adjusted for sampling weights as prescribed by the NSSO. For brevity, the beta coefficients and standard errors (SE) for the fully adjusted linear regression models and odds ratios and confidence interval (at 95\%) for logistic regression model are reported. We also estimate the variance partition coefficient for both set of regressions to highlight the between-occupational group differences in calorie deprivations (Browne et al., 2005, Goldstein et al 2002). Using the estimated variance of random effects, the variance partition coefficients (VPCs) at each level for the respective models (variation in calorie intake or variation in the log odds of receiving insufficient caloric intake) is computed. The VPC for the concerned level is calculated by dividing the estimated variance at the concerned level by the total variance. While calculating the total variance in the logistic regression, a latent variable methods approach is used whereby the between-household variance is assumed to follow a standard logistic distribution with a value of $3.29[33,34]$. The regression analysis is adjusted for the following indicators of household socioeconomic status: age and sex of household head, household size, and education of household head, religion, social group, wealth quintile, sampling weights and standard errors clustered at the district and state level. The wealth quintiles are constructed using principle component analysis (PCA) on the 20 household durable items from NSS 68 Schedule 1.0. The analysis is performed in Stata 15.0 using runm/win module [35-37].

\section{Results}

The average per capita calorie intake is very similar across rural $(2172 \mathrm{kcal})$ and urban $(2163 \mathrm{kcal})$ India (Table 2). In rural areas, households under high level services and managerial professions (particularly science, life science and health professionals) report the highest average per capita calorie intake (in excess of $2300 \mathrm{kcal})$. The lowest calorie intake (2086 kcal) is estimated for agricultural and fishery labor 
households. In urban areas, the similar group of professionals and managers has highest levels of caloric intake (in excess of $2400 \mathrm{kcal}$ ) whereas households belonging to low-end workers and laborers report low intake (below $2100 \mathrm{kcal}$ ). It is worth noting that across both rural and urban areas, market oriented skilled agricultural and fishery workers' (2261 kcal - rural, $2165 \mathrm{kcal}$ - urban) have higher calorie intakes than those belonging to subsistence agricultural (2165 kcal - rural, $2149 \mathrm{kcal}$ - urban) and fishery workers or agricultural and fishery laborer (2086 kcal - rural, $2071 \mathrm{kcal}$ - urban). Nevertheless, across both rural and urban areas, the calorie intake has a wider distribution around the mean and can be confirmed by glancing through the box-plots presented in Fig. 1 or at the standard deviations reported in Table 2. Further, Table 2 also reports the percentage of households with insufficient per capita calorie intake is $33.8 \%$ in rural India and $18.5 \%$ in urban India. This percentage varied significantly across occupational groups. For instance, in rural India the highest level of insufficiencies is noted among workers in precision, handicraft, printing and related trades ( $45.2 \%$ households). Agricultural and fishery laborer as well as extraction workers also display higher levels of caloric deprivation (39\% households). In urban areas, caloric deprivations are highly concentrated among households engaged in elementary occupation related to sales and services ( $27 \%$ households) whereas legislators, professionals and managers have very low estimated prevalence of caloric deprivation. 
Table 2

Average per capita calories consumed per day per household and the number and percentage of households with insufficient calorie intake among a nationally representative sample of households, National Sample Survey, India 2011-2012.

\begin{tabular}{|c|c|c|c|c|c|c|c|c|}
\hline \multirow[t]{3}{*}{$\begin{array}{l}\text { Household occupational groups } \\
\text { (NCO) }\end{array}$} & \multicolumn{4}{|c|}{$\begin{array}{l}\text { Calorie intake per Capita per } \\
\text { Household }\end{array}$} & \multicolumn{4}{|c|}{ \% Undernourished Households } \\
\hline & \multicolumn{2}{|l|}{ Rural } & \multicolumn{2}{|l|}{ Urban } & \multicolumn{2}{|c|}{ Rural } & \multicolumn{2}{|c|}{ Urban } \\
\hline & Mean & SD & Mean & SD & $\%$ & $\mathrm{n}$ & $\%$ & $n$ \\
\hline Legislators \& senior officials & 2382 & 793 & 2490 & 787 & 26.3 & 33 & 5.7 & 14 \\
\hline Corporate managers & 2176 & 598 & 2155 & 553 & 33.8 & 1,314 & 16.5 & 897 \\
\hline General managers & 2377 & 595 & 2594 & 766 & 26.4 & 10 & 5.4 & 7 \\
\hline Science professionals & 2420 & 578 & 2489 & 700 & 18.8 & 24 & 8.8 & 69 \\
\hline $\begin{array}{l}\text { Life science \& health } \\
\text { professionals }\end{array}$ & 2453 & 1158 & 2605 & 758 & 27.0 & 63 & 3.3 & 17 \\
\hline Teaching professionals & 2294 & 618 & 2364 & 612 & 28.6 & 264 & 8.8 & 92 \\
\hline Other professionals & 2226 & 576 & 2233 & 593 & 27.9 & 310 & 17.2 & 251 \\
\hline Science associate professionals & 2200 & 644 & 2338 & 680 & 34.9 & 45 & 10.8 & 49 \\
\hline $\begin{array}{l}\text { Life science \& health associate } \\
\text { professionals }\end{array}$ & 2321 & 543 & 2277 & 619 & 22.0 & 52 & 14.2 & 49 \\
\hline $\begin{array}{l}\text { Teaching associate } \\
\text { professionals }\end{array}$ & 2267 & 592 & 2318 & 690 & 28.0 & 540 & 13.1 & 109 \\
\hline Other associate professionals & 2275 & 629 & 2296 & 651 & 23.5 & 195 & 13.0 & 169 \\
\hline Office clerks & 2306 & 708 & 2243 & 1147 & 23.3 & 263 & 15.3 & 234 \\
\hline Customer services clerks & 2193 & 444 & 2224 & 572 & 30.4 & 39 & 12.5 & 38 \\
\hline $\begin{array}{l}\text { Personal \& protective service } \\
\text { workers }\end{array}$ & 2172 & 607 & 2222 & 613 & 34.9 & 644 & 16.7 & 403 \\
\hline $\begin{array}{l}\text { Models, sales persons \& } \\
\text { demonstrators }\end{array}$ & 2123 & 568 & 2110 & 630 & 38.2 & 1,238 & 19.8 & 798 \\
\hline $\begin{array}{l}\text { Market oriented skilled } \\
\text { agricultural \& fishery workers }\end{array}$ & 2261 & 634 & 2165 & 601 & 27.5 & 4,388 & 18.0 & 323 \\
\hline $\begin{array}{l}\text { Subsistence agricultural \& } \\
\text { fishery workers }\end{array}$ & 2165 & 540 & 2149 & 308 & 33.9 & 175 & 4.6 & 14 \\
\hline $\begin{array}{l}\text { Extraction \& building trades } \\
\text { workers }\end{array}$ & 2096 & 581 & 2020 & 508 & 39.2 & 1,652 & 22.8 & 661 \\
\hline
\end{tabular}

Source: Data from National Sample Survey, Government of India 


\begin{tabular}{|c|c|c|c|c|c|c|c|c|}
\hline \multirow{3}{*}{$\begin{array}{l}\text { Household occupational groups } \\
\text { (NCO) } \\
\text { Metal, machinery \& related } \\
\text { trades workers }\end{array}$} & \multicolumn{4}{|c|}{$\begin{array}{l}\text { Calorie intake per Capita per } \\
\text { Household }\end{array}$} & \multicolumn{4}{|c|}{ \% Undernourished Households } \\
\hline & \multicolumn{2}{|l|}{ Rural } & \multicolumn{2}{|l|}{ Urban } & \multicolumn{2}{|l|}{ Rural } & \multicolumn{2}{|c|}{ Urban } \\
\hline & 2168 & 578 & 2146 & 666 & 34.8 & 301 & 19.6 & 288 \\
\hline $\begin{array}{l}\text { Precision, handicraft, printing \& } \\
\text { related trades workers }\end{array}$ & 2025 & 467 & 2119 & 585 & 45.2 & 151 & 20.3 & 109 \\
\hline $\begin{array}{l}\text { Other craft \& related trades } \\
\text { workers }\end{array}$ & 2099 & 568 & 2107 & 575 & 38.1 & 616 & 19.4 & 326 \\
\hline $\begin{array}{l}\text { Stationary plant \& related } \\
\text { operators }\end{array}$ & 2134 & 586 & 2146 & 578 & 33.5 & 106 & 21.2 & 50 \\
\hline $\begin{array}{l}\text { Machine operators \& } \\
\text { assemblers }\end{array}$ & 2155 & 548 & 2196 & 552 & 34.7 & 204 & 16.7 & 189 \\
\hline Drivers \& mobile-plant operators & 2146 & 564 & 1991 & 473 & 35.3 & 775 & 26.0 & 464 \\
\hline $\begin{array}{l}\text { Sales \& services elementary } \\
\text { occupations }\end{array}$ & 2128 & 579 & 2054 & 559 & 36.3 & 408 & 26.7 & 505 \\
\hline $\begin{array}{l}\text { Agricultural, fishery \& related } \\
\text { labourers }\end{array}$ & 2086 & 520 & 2071 & 504 & 39.3 & 2,215 & 21.3 & 215 \\
\hline $\begin{array}{l}\text { Mining, construction, } \\
\text { manufacturing \& transport } \\
\text { labourers }\end{array}$ & 2116 & 535 & 2110 & 630 & 38.8 & 2,009 & 23.9 & 707 \\
\hline All households & 2172 & 590 & 2163 & 636 & 33.8 & 18034 & 18.5 & 7047 \\
\hline
\end{tabular}

Among rural households, multilevel linear regression estimates (Table 3 ) indicate that compared to agricultural and fishery laborers, households of legislators and senior officials, life science and health professionals, and market oriented skilled agricultural and fishery workers have significantly higher average per capita calorie intake. While a number of other service sector professionals depict higher household calorie intake but the differences are not statistically significant. Among urban households, a large number of households from service sector background as well as those engaged in market oriented skilled agricultural and fishery work report significantly higher levels of calorie intake. There is no significant difference in calorie intake of low-end occupations and laborers in urban or rural areas. Further in Table 3, we also present the multilevel logistic regression estimates for the association of caloric intake and occupational background while adjusting for demographic and socioeconomic factors such as age and sex of household health, household size, and education of household head, religion, social group, and household wealth quintile. The estimates suggest that in rural areas, only households engaged in market oriented skilled agricultural and fishery works have significantly (at $5 \%$ level) lower odds ratio (OR: 0.72 , with $95 \% \mathrm{Cl}: 0.63 ; 0.82$ ) of having insufficient calorie intake compared to the agricultural and fishery laborer households. While households with professionals and managers also depict lower odds but the 
effects are significant only at $10 \%$ level. In urban areas, a similar relative advantage is discernible for market orientation and skills among agricultural and fishery worker households (OR: 0.72, with 95\% Cl: $0.59 ; 0.86)$. The odds of receiving insufficient calorie intake are also much lower for the service sector professionals, particularly life science and health professionals (OR: 0.33 , with $95 \% \mathrm{Cl}: 0.13 ; 0.81$ ). However, households engaged in sales and services based elementary occupations (OR: 1.35, with 95\% Cl: $1.04 ; 1.75)$ are $35 \%$ more likely to have insufficient caloric intake compared to the agricultural and fishery laborer households. 
Table 3

Multilevel linear and logistic regression estimates for the association of household occupational group with per capita calorie consumption per day per household and having insufficient caloric intake in rural and urban India, National Sample Survey, 2011-2012.

\begin{tabular}{|c|c|c|c|c|c|c|c|c|}
\hline \multirow{3}{*}{$\begin{array}{l}\text { Household } \\
\text { occupational groups } \\
\text { (NCO) }\end{array}$} & \multicolumn{4}{|c|}{$\begin{array}{l}\text { Calorie Consumption per Capita per } \\
\text { Household }\end{array}$} & \multicolumn{4}{|c|}{$\begin{array}{l}\text { Households having } \\
\text { insufficient caloric intake }\end{array}$} \\
\hline & \multicolumn{2}{|l|}{ Rural } & \multicolumn{2}{|l|}{ Urban } & \multicolumn{2}{|l|}{ Rural } & \multicolumn{2}{|l|}{ Urban } \\
\hline & Coef & SE & Coef & SE & AOR & $\begin{array}{l}95 \% \\
\mathrm{Cl}\end{array}$ & AOR & $\begin{array}{l}95 \% \\
\mathrm{Cl}\end{array}$ \\
\hline $\begin{array}{l}\text { Agricultural, fishery \& } \\
\text { related labourers }\end{array}$ & ref & - & ref & - & ref & - & ref & - \\
\hline $\begin{array}{l}\text { Legislators \& senior } \\
\text { officials }\end{array}$ & $277.3^{\star \star *}$ & 88.4 & $185.0 * \star \star$ & 54.8 & $0.56^{*}$ & $\begin{array}{l}{[0.29} \\
1.09]\end{array}$ & $0.50 *$ & $\begin{array}{l}{[0.24 ;} \\
1.06]\end{array}$ \\
\hline Corporate managers & 26.8 & 17.9 & $63.7^{\star \star}$ & 30.9 & 0.94 & $\begin{array}{l}{[0.80 ;} \\
1.12]\end{array}$ & 0.91 & $\begin{array}{l}{[0.69 ;} \\
1.20]\end{array}$ \\
\hline General managers & 91.1 & 94.4 & $303.4^{\star \star \star}$ & 79.7 & 1.36 & $\begin{array}{l}{[0.46} \\
4.00]\end{array}$ & 0.50 & $\begin{array}{l}{[0.20 ;} \\
1.21]\end{array}$ \\
\hline Science professionals & 109.3 & 73.3 & $200.4^{\star \star \star}$ & 32.2 & $0.58^{*}$ & $\begin{array}{l}{[0.31 ;} \\
1.07]\end{array}$ & 0.79 & $\begin{array}{l}{[0.50 ;} \\
1.24]\end{array}$ \\
\hline $\begin{array}{l}\text { Life science \& health } \\
\text { professionals }\end{array}$ & $178.4^{\star \star}$ & 86.1 & $299.2^{\star \star \star}$ & 35.6 & 0.90 & $\begin{array}{l}{[0.53} \\
1.53]\end{array}$ & $0.33^{\star \star}$ & $\begin{array}{l}{[0.13 ;} \\
0.81]\end{array}$ \\
\hline $\begin{array}{l}\text { Teaching } \\
\text { professionals }\end{array}$ & 16.8 & 38.2 & $110.5^{\star \star \star}$ & 28.9 & 1.06 & $\begin{array}{l}{[0.81} \\
1.39]\end{array}$ & $0.68 *$ & $\begin{array}{l}{[0.45 ;} \\
1.03]\end{array}$ \\
\hline Other professionals & 6.7 & 24.1 & 39.5 & 26.8 & 0.91 & $\begin{array}{l}{[0.72 ;} \\
1.16]\end{array}$ & 1.16 & $\begin{array}{l}{[0.88 ;} \\
1.55]\end{array}$ \\
\hline $\begin{array}{l}\text { Science associate } \\
\text { professionals }\end{array}$ & 7.8 & 72.8 & $87.8^{\star}$ & 52.1 & 0.95 & $\begin{array}{l}{[0.57} \\
1.58]\end{array}$ & 0.71 & $\begin{array}{l}{[0.41 ;} \\
1.22]\end{array}$ \\
\hline $\begin{array}{l}\text { Life science \& health } \\
\text { associate } \\
\text { professionals }\end{array}$ & 66.9 & 55.9 & $72.4^{\star}$ & 43.8 & 0.74 & $\begin{array}{l}{[0.43} \\
1.29]\end{array}$ & 0.92 & $\begin{array}{l}{[0.60 ;} \\
1.41]\end{array}$ \\
\hline $\begin{array}{l}\text { Teaching associate } \\
\text { professionals }\end{array}$ & 33.3 & 24.6 & $75.7 \star \star$ & 31.9 & 0.96 & $\begin{array}{l}{[0.78 ;} \\
1.17]\end{array}$ & 1.03 & $\begin{array}{l}{[0.71 ;} \\
1.49]\end{array}$ \\
\hline $\begin{array}{l}\text { Other associate } \\
\text { professionals }\end{array}$ & $60.3^{*}$ & 35.3 & $85.2^{\star \star}$ & 33.1 & 0.81 & $\begin{array}{l}{[0.60 ;} \\
1.08]\end{array}$ & 0.95 & $\begin{array}{l}{[0.70 ;} \\
1.29]\end{array}$ \\
\hline
\end{tabular}

${ }^{*} p<0.05,{ }^{* *} p<0.01,{ }^{* \star} p<0.001$

Notes: The models are adjusted for age and sex of household head, household size, education of household head, religion, social group, wealth quintile, sampling weights and standard errors clustered at the district and state level. 


\begin{tabular}{|c|c|c|c|c|c|c|c|c|}
\hline \multirow{3}{*}{$\begin{array}{l}\text { Household } \\
\text { occupational groups } \\
\text { (NCO) }\end{array}$} & \multicolumn{4}{|c|}{$\begin{array}{l}\text { Calorie Consumption per Capita per } \\
\text { Household }\end{array}$} & \multicolumn{4}{|c|}{$\begin{array}{l}\text { Households having } \\
\text { insufficient caloric intake }\end{array}$} \\
\hline & \multicolumn{2}{|l|}{ Rural } & \multicolumn{2}{|l|}{ Urban } & \multicolumn{2}{|l|}{ Rural } & \multicolumn{2}{|l|}{ Urban } \\
\hline & Coef & SE & Coef & SE & AOR & $\begin{array}{l}95 \% \\
\mathrm{Cl}\end{array}$ & AOR & $\begin{array}{l}95 \% \\
\mathrm{Cl}\end{array}$ \\
\hline Office clerks & 54.1 & 36.2 & 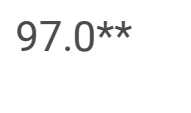 & 47.5 & $0.77 *$ & $\begin{array}{l}{[0.57 ;} \\
1.03]\end{array}$ & 1.03 & $\begin{array}{l}{[0.74 ;} \\
1.44]\end{array}$ \\
\hline $\begin{array}{l}\text { Customer services } \\
\text { clerks }\end{array}$ & -74.4 & 66.9 & 49.9 & 42.2 & 1.08 & $\begin{array}{l}{[0.48 ;} \\
2.42]\end{array}$ & 0.99 & $\begin{array}{l}{[0.59 ;} \\
1.67]\end{array}$ \\
\hline $\begin{array}{l}\text { Personal \& protective } \\
\text { service workers }\end{array}$ & 21.3 & 25.2 & 55.1 & 35.6 & 1.00 & $\begin{array}{l}{[0.80} \\
1.25]\end{array}$ & 0.99 & $\begin{array}{l}{[0.71 ;} \\
1.38]\end{array}$ \\
\hline $\begin{array}{l}\text { Models, sales persons } \\
\& \text { demonstrators }\end{array}$ & 1.8 & 26.9 & 10.9 & 32.0 & 1.08 & $\begin{array}{l}{[0.91 ;} \\
1.28]\end{array}$ & 1.11 & $\begin{array}{l}{[0.83 ;} \\
1.49]\end{array}$ \\
\hline $\begin{array}{l}\text { Market oriented skilled } \\
\text { agricultural \& fishery } \\
\text { workers }\end{array}$ & $122.2^{\star \star \star}$ & 19.2 & $137.4^{\star \star \star}$ & 29.0 & $0.72^{\star \star \star}$ & $\begin{array}{l}{[0.63 ;} \\
0.82]\end{array}$ & $0.72^{\star \star \star}$ & $\begin{array}{l}{[0.59 ;} \\
0.86]\end{array}$ \\
\hline $\begin{array}{l}\text { Subsistence } \\
\text { agricultural \& fishery } \\
\text { workers }\end{array}$ & 3.4 & 31.9 & 29.6 & 35.4 & 1.10 & $\begin{array}{l}{[0.80 ;} \\
1.52]\end{array}$ & 0.97 & $\begin{array}{l}{[0.42 ;} \\
2.20]\end{array}$ \\
\hline $\begin{array}{l}\text { Extraction \& building } \\
\text { trades workers }\end{array}$ & -3.8 & 19.7 & 8.8 & 26.3 & 1.02 & $\begin{array}{l}{[0.86 ;} \\
1.21]\end{array}$ & 1.01 & $\begin{array}{l}{[0.78 ;} \\
1.31]\end{array}$ \\
\hline $\begin{array}{l}\text { Metal, machinery \& } \\
\text { related trades workers }\end{array}$ & 10.4 & 30.3 & 20.7 & 33.1 & 1.03 & $\begin{array}{l}{[0.84 ;} \\
1.27]\end{array}$ & 1.13 & $\begin{array}{l}{[0.80 ;} \\
1.62]\end{array}$ \\
\hline $\begin{array}{l}\text { Precision, handicraft, } \\
\text { printing \& related } \\
\text { trades workers }\end{array}$ & -63.5 & 45.0 & -23.2 & 59.0 & 1.40 & $\begin{array}{l}{[0.87} \\
2.26]\end{array}$ & 1.21 & $\begin{array}{l}{[0.89 ;} \\
1.66]\end{array}$ \\
\hline $\begin{array}{l}\text { Other craft \& related } \\
\text { trades workers }\end{array}$ & -15.4 & 27.0 & 43.1 & 34.2 & 1.08 & $\begin{array}{l}{[0.90 ;} \\
1.30]\end{array}$ & 0.87 & $\begin{array}{l}{[0.63 ;} \\
1.22]\end{array}$ \\
\hline $\begin{array}{l}\text { Stationary plant \& } \\
\text { related operators }\end{array}$ & -0.5 & 40.7 & 31.1 & 40.7 & 0.93 & $\begin{array}{l}{[0.54} \\
1.60]\end{array}$ & 1.10 & $\begin{array}{l}{[0.71 ;} \\
1.71]\end{array}$ \\
\hline $\begin{array}{l}\text { Machine operators \& } \\
\text { assemblers }\end{array}$ & 38.1 & 39.2 & $51.5^{\star \star}$ & 25.5 & 0.94 & $\begin{array}{l}{[0.68 ;} \\
1.30]\end{array}$ & 0.99 & $\begin{array}{l}{[0.74 ;} \\
1.33]\end{array}$ \\
\hline $\begin{array}{l}\text { Drivers \& mobile-plant } \\
\text { operators }\end{array}$ & 21.9 & 38.4 & -0.2 & 31.1 & 0.90 & $\begin{array}{l}{[0.70 ;} \\
1.17]\end{array}$ & 1.12 & $\begin{array}{l}{[0.83 ;} \\
1.51]\end{array}$ \\
\hline \multicolumn{9}{|c|}{${ }^{*} p<0.05,{ }^{* \star} p<0.01,{ }^{* \star \star} p<0.001$} \\
\hline \multicolumn{9}{|c|}{$\begin{array}{l}\text { Notes: The models are adjusted for age and sex of household head, household size, education of } \\
\text { household head, religion, social group, wealth quintile, sampling weights and standard errors } \\
\text { clustered at the district and state level. }\end{array}$} \\
\hline \multicolumn{9}{|c|}{ AOR - Adjusted Odds Ratio } \\
\hline
\end{tabular}




\begin{tabular}{|c|c|c|c|c|c|c|c|c|}
\hline \multirow{3}{*}{$\begin{array}{l}\text { Household } \\
\text { occupational groups } \\
\text { (NCO) }\end{array}$} & \multicolumn{4}{|c|}{$\begin{array}{l}\text { Calorie Consumption per Capita per } \\
\text { Household }\end{array}$} & \multicolumn{4}{|c|}{$\begin{array}{l}\text { Households having } \\
\text { insufficient caloric intake }\end{array}$} \\
\hline & \multicolumn{2}{|l|}{ Rural } & \multicolumn{2}{|l|}{ Urban } & \multicolumn{2}{|l|}{ Rural } & \multicolumn{2}{|l|}{ Urban } \\
\hline & Coef & SE & Coef & SE & AOR & $\begin{array}{l}95 \% \\
\mathrm{Cl}\end{array}$ & AOR & $\begin{array}{l}95 \% \\
\mathrm{Cl}\end{array}$ \\
\hline $\begin{array}{l}\text { Sales \& services } \\
\text { elementary } \\
\text { occupations }\end{array}$ & -3.5 & 27.0 & -19.8 & 29.6 & 0.98 & $\begin{array}{l}{[0.80} \\
1.21]\end{array}$ & $1.35^{\star \star}$ & $\begin{array}{l}{[1.04 ;} \\
1.75]\end{array}$ \\
\hline $\begin{array}{l}\text { Mining, construction, } \\
\text { manufacturing \& } \\
\text { transport labourers }\end{array}$ & -6.2 & 18.9 & 25.1 & 28.2 & 1.11 & $\begin{array}{l}{[0.94} \\
1.30]\end{array}$ & 1.03 & $\begin{array}{l}{[0.83 ;} \\
1.28]\end{array}$ \\
\hline \multicolumn{9}{|c|}{${ }^{*} p<0.05,{ }^{* *} p<0.01,{ }^{* \star *} p<0.001$} \\
\hline \multicolumn{9}{|c|}{$\begin{array}{l}\text { Notes: The models are adjusted for age and sex of household head, household size, education of } \\
\text { household head, religion, social group, wealth quintile, sampling weights and standard errors } \\
\text { clustered at the district and state level. }\end{array}$} \\
\hline \multicolumn{9}{|c|}{ AOR - Adjusted Odds Ratio } \\
\hline
\end{tabular}

For rural and urban India, Table 4 presents the variance partition coefficients (VPC) for the multilevel linear regression model for average per capita calorie intake and multilevel logistic regression model for households having insufficient caloric intake. The models use five levels wherein the nesting runs in a hierarchical manner starting from households, occupational groups, districts, region and state of residence. The VPC can reveal the between-group variations attributable at the various levels. In this regard, the null model for average per capita calorie consumption in rural India shows that $10.2 \%$ of the total variance in this indicator is attributable to differences in occupational groups whereas state-related differences account for $7.4 \%$ of the variation in calorie intake. After adjusting of demographic and socioeconomic factors, the VPC of occupational groups declines to $7.8 \%$. However, in urban areas, a greater proportion of variability in calorie intake is attributable to occupational group related differences (VPC 18.1\% null model; VPC 14.8\% fully-adjusted model). The geographic boundaries of states and districts have low relevance in explaining variability across urban areas. In fact, region of residence has very low relevance in explaining variations in either outcome across rural or urban India. Further, the VPCs from logistic regression for households having insufficient caloric intake also present similar insights. Finally, the occupational group-specific random intercepts from the four respective null models are plotted in Fig. 2. It is inferred that in rural India caloric intake of about two-thirds of the occupational groups cannot be distinguished from the overall average (Fig. 2a). However, more significant differences are apparent in urban areas (Fig. 2b). In particular, most of the legislators, professionals and managers have a higher average intake. Figure $2 \mathrm{c}$ and $2 \mathrm{~d}$ reveal that households engaged in mining, construction, manufacturing and transport labor activities are at an elevated risk of insufficient caloric intake. 
Table 4

Variance estimates and variance partition coefficients [in parenthesis] for multilevel linear (standard error) and logit regressions (95\% Cl) at occupational group-, district-, region- and state-levels from null and adjusted models (National Sample Survey, India 2011-12)

\begin{tabular}{|c|c|c|c|c|}
\hline \multirow[t]{3}{*}{ Level } & \multicolumn{4}{|c|}{ Calorie Consumption per Capita per Household } \\
\hline & \multicolumn{2}{|l|}{ Rural India } & \multicolumn{2}{|l|}{ Urban India } \\
\hline & Null model & $\begin{array}{l}\text { Fully adjusted } \\
\text { model* }\end{array}$ & Null model & $\begin{array}{l}\text { Fully adjusted } \\
\text { model* }\end{array}$ \\
\hline \multirow[t]{2}{*}{ State } & $28904[7.4 \%]$ & $36781[10.8 \%]$ & $21361[4.4 \%]$ & $20100[5.3 \%]$ \\
\hline & $(10340)$ & $(8762)$ & (8448) & (5963) \\
\hline \multirow[t]{2}{*}{ Region } & $9777[2.5 \%]$ & 8056 [2.4\%] & $8173[1.7 \%]$ & 7577 [2.0\%] \\
\hline & $(2546)$ & $(2060)$ & (3105) & $(2772)$ \\
\hline \multirow[t]{2}{*}{ District } & $20352[5.2 \%]$ & $20560[6.0 \%]$ & $17541[3.6 \%]$ & 18703 [4.9\%] \\
\hline & $(3665)$ & $(3630)$ & $(2800)$ & $(3122)$ \\
\hline \multirow[t]{2}{*}{$\begin{array}{l}\text { NCO Occupational } \\
\text { group }\end{array}$} & $\begin{array}{l}39954 \\
{[10.2 \%]}\end{array}$ & $26386[7.8 \%]$ & $\begin{array}{l}88193 \\
{[18.1 \%]}\end{array}$ & $56244[14.8 \%]$ \\
\hline & $(3467)$ & $(2971)$ & $(23976)$ & (19608) \\
\hline \multirow[t]{2}{*}{ Household } & $\begin{array}{l}293923 \\
{[74.8 \%]}\end{array}$ & $248559[73.0 \%]$ & $\begin{array}{l}352001 \\
{[72.2 \%]}\end{array}$ & $276570[72.9 \%]$ \\
\hline & (31788) & (28095) & (38399) & (37475) \\
\hline \multirow[t]{3}{*}{ Level } & \multicolumn{4}{|c|}{ Households with insufficient caloric intake } \\
\hline & \multicolumn{2}{|l|}{ Rural India } & \multicolumn{2}{|l|}{ Urban India } \\
\hline & Null model & $\begin{array}{l}\text { Fully adjusted } \\
\text { model* }\end{array}$ & Null model & $\begin{array}{l}\text { Fully adjusted } \\
\text { model* }^{*}\end{array}$ \\
\hline \multirow[t]{2}{*}{ State } & $0.216[5.0 \%]$ & $0.409[9.2 \%]$ & $0.064[1.5 \%]$ & $0.149[3.4 \%]$ \\
\hline & $\begin{array}{l}(0.048 ; \\
0.384)\end{array}$ & $(0.202 ; 0.615)$ & $\begin{array}{l}(-0.015 \\
0.142)\end{array}$ & $(0.024 ; 0.274)$ \\
\hline \multirow[t]{2}{*}{ Region } & $0.145[3.4 \%]$ & $0.143[3.2 \%]$ & $0.216[4.9 \%]$ & $0.214[4.9 \%]$ \\
\hline & $\begin{array}{l}(0.056 ; \\
0.233)\end{array}$ & $(0.04 ; 0.245)$ & $\begin{array}{l}(0.081 ; \\
0.352)\end{array}$ & $(0.063 ; 0.364)$ \\
\hline District & $0.253[5.9 \%]$ & $0.281[6.3 \%]$ & $0.329[7.5 \%]$ & $0.376[8.5 \%]$ \\
\hline
\end{tabular}

*The models are adjusted for age and sex of household head, household size, education of household head, religion, social group, wealth quintile, place of residence, sampling weights and standard errors clustered at the NCO occupational group, district, region and state level. 


\begin{tabular}{|c|c|c|c|c|}
\hline \multirow[t]{3}{*}{ Level } & \multicolumn{4}{|c|}{ Calorie Consumption per Capita per Household } \\
\hline & \multicolumn{2}{|l|}{ Rural India } & \multicolumn{2}{|l|}{ Urban India } \\
\hline & Null model & $\begin{array}{l}\text { Fully adjusted } \\
\text { mode|* }\end{array}$ & Null model & $\begin{array}{l}\text { Fully adjusted } \\
\text { model* }^{\star}\end{array}$ \\
\hline & $\begin{array}{l}(0.189 ; \\
0.316)\end{array}$ & $(0.216 ; 0.346)$ & $\begin{array}{l}(0.249 ; \\
0.409)\end{array}$ & $(0.269 ; 0.483)$ \\
\hline \multirow{2}{*}{$\begin{array}{l}\text { NCO Occupational } \\
\text { group }\end{array}$} & $0.380[8.9 \%]$ & $0.315[7.1 \%]$ & $0.483[11.0 \%]$ & $0.381[8.6 \%]$ \\
\hline & $\begin{array}{l}(0.291 ; \\
0.469)\end{array}$ & $(0.233 ; 0.398)$ & $\begin{array}{l}(0.294 ; \\
0.672)\end{array}$ & $(0.185 ; 0.577)$ \\
\hline $\begin{array}{l}\text { *The models are ad } \\
\text { household head, rel } \\
\text { standard errors clus }\end{array}$ & $\begin{array}{l}\text { for age and } \\
\text { social group, } \\
\text { at the NCO or }\end{array}$ & $\begin{array}{l}\text { of household } \\
\text { alth quintile, pla } \\
\text { Jational group, }\end{array}$ & $\begin{array}{l}\text { usehold size, } e \\
\text { sidence, samp } \\
\text { region and sta }\end{array}$ & $\begin{array}{l}\text { ucation of } \\
\text { ig weights and } \\
\text { level. }\end{array}$ \\
\hline
\end{tabular}

\section{Discussion And Conclusion}

This analysis of nationally representative survey across India (2011-12) finds that the patterns of caloric intake and deprivations are significantly associated with occupational background of the households among both rural and urban households. Households dependent on occupations such as casual labour in agricultural and non-agricultural activities as well as those involved in low-end sales and services consumed fewer calories than others and also were at an elevated risk of caloric deprivation. In contrast, households engaged in market oriented skilled agricultural and fishery as well as the high-level professionals and managers had more than adequate calorie consumption and also were at lowest risk of such deprivations. These patterns mirror the evidence on disproportionate burden of poverty and deprivation among unskilled agricultural and non-agricultural workers in rural and urban India $[13,38,17$, $18,39-40]$.

The regressions, particularly the occupation-specific random effects (Figure 2), reveal that market oriented skilled agricultural and fishery workers are among the select few occupations which display robust association with food and nutritional security. The households belonging to this occupational category display significantly higher levels of calorie consumption and also a lower risk of caloric deprivation. The nutritional well-being of this group is matched only by households belonging to legislators, professionals and managers. The occupation-specific random-intercepts, confirm the stark inter-occupational disparities in nutritional intake with highest disadvantage for unskilled mining and construction laborers as well as those engaged in elementary sales and services workers. The betweenoccupation differences are also higher in urban areas. This is an important concern because higher degree of occupational diversification has not led to more equitable nutritional intake although there is evidence to suggest its favorable influence on poverty reduction [41]. 
Conventionally, poverty and nutritional deprivation in India is largely discussed as a state-level phenomenon $[42-47,10]$. However, there is limited evidence to understand whether it is more associated with occupational differences or other forms of disparities across states and regions. In this regard, the variance partition coefficients (VPCs) provide an overwhelming evidence to emphasize on occupations approach towards poverty and nutritional well-being. The VPCs highlight that occupational groups have the greatest effects on caloric intake across households and these effects were greater than the statelevel influence. In particular, it is noted that the amount of variation in caloric intake attributable to the occupations ( $10.2 \%$ and $18.1 \%$ for calorie consumption in rural and urban India, respectively) is substantial even when adjusted for standard household-level socioeconomic correlates. These insights when combined with the occupation-specific random-effects suggest that policy focus to promote market oriented skilled agricultural and fishery workers can be an equally good option as direct investments in professional and managerial skills for manufacturing and services. Moreover, these findings reiterate the need for a balanced approach towards skill development in India whereby a focus on agricultural sector is not undermined because of narratives favoring manufacturing and services.

Yet, the success of skill development and training programmes depends on a range of factors. In particular, occupational mobility declines with age, therefore it is important to impart skill and training at the right age to increase the output of skill development programmes. As such, in 2010, for the first time the share of working age population in India increased to $60 \%$ of the total population [48]. Such favorable population age-structure is expected to have significant impact on economic growth and development [50-53]. However, despite such favorable outlook, the developmental narrative for India is less optimistic largely because of a number of growth constraints. In particular, inadequate labor skills and low employment opportunities are regarded as a major challenge for higher growth and sustainable development in India [54-56]. Given the challenges, recent policies have emphasized on skill development to realize the full demographic dividend. In particular, the skill development campaign with major initiatives like Pradhan Mantri Kaushal Vikas Yojana (2015) and Kaushal and Rozgar Mela (2016) are apt examples of specific policy interests. But a skill-based approach is more likely to favor the younger generations whereas these policies also have to accommodate the needs and concerns associated with cohorts of older adults. In the absence on an all-encompassing approaching, it is likely that poverty and deprivation can turn out to be an inter-generational affair whereby only the young generation within poor households is presented with any potential chances to improve upon household well-being.

Furthermore, it is equally important to entail a gendered-perspective for skill development. In fact, there are success stories (such as Kudumbshree, the Kerala State Poverty Eradication Mission) to demonstrate that a gendered-approach towards market oriented and skilled agrarian workers can be an effective approach to enhance the income and nutritional status of households $[57,58]$. This also implies that the Skill India agenda should focus on mainstreaming agrarian occupations by promoting professional and technical education among those engaged in unskilled and subsistence agriculture and fishery. In particular, despite a vast coastline, the fishery sector in India is relatively unexplored for its potential impact on nutritional and income security. It is no surprise that the policy paradigm is rather in sync with the developing world whereby poverty among small-scale fisheries has remained a neglected aspect of 
development $[15,59,60,38,61]$. Whereas, there is increasing evidence to support that modernization of the fisheries sector offers tremendous potential for development and growth [62 - 65].

Nevertheless, in concluding, it is worth noting the three limitations of the analysis that can be largely associated with the nature of survey and the data. First, given the cross-sectional design, the results do not necessarily reveal the casual direction of association between occupation and caloric intake even though this does not impact the results regarding occupation-specific disparities and advantages in caloric intake. Second, although the NCO 2004 classification is sufficiently disaggregated to arrive at some meaningful inferences but further disaggregation is advisable to understand the intricacies associated with skilled occupations within agriculture and fishery sectors. In fact, in the survey the NCO 2004 codes are missing for about 7.3\% households and this can have a certain influence on the relative significance of the estimates. Third, the outcome indicator of household calorie consumption does not provide adequate insights regarding individual-level differences. Besides, to some extent, this indicator marginally underestimates the total calorie intake because of non-inclusion of food consumed outside the home [66].

\section{Abbreviations}

HCES - Household Consumer Expenditure Survey

NSS - National Sample Survey

MSDE - Ministry of skill Development and Entrepreneurship

MOSPI - Ministry of skill Development and Programme Implementation

SE - Standard Error

VPC - Variance Partition Coefficients

PCA - Principle Component Analysis

OR - Odds Ratio

$\mathrm{Cl}$ - Confidence Interval

\section{Declarations}

Ethics approval and consent to participate: Not Applicable

Consent for publication: Not Applicable

Availability of data and material: The data is available publicly without any legal or ethical restrictions at official website of MOSPI: http://www.mospi.gov.in/ 
Competing interests: The authors declare that they have no competing interests

Funding: No funding was received

Authors' contributions: All authors contributed equally.

Acknowledgements: Not Applicable

\section{References}

1. Lewis A. The Theory of Economic Growth, R.D. Irwin. Homewood, Illinois, 1955.

2. Dercon S, Gollin D. Agriculture in African development: theories and strategies. Annu Rev Resour Econ. 2014;6(1):471-92.

3. Bryceson DF. Deagrarianization and rural employment in sub-Saharan Africa: a sectoral perspective. World Dev. 1996;24(1):97-111.

4. Cervantes-Godoy D, Dewbre J Economic Importance of Agriculture for Poverty Reduction. OECD Food, Agriculture and Fisheries Working Papers, No. 23, OECD Publishing, 2010.

5. Byerlee D, De Janvry A, Sadoulet E. Agriculture for development: Toward a new paradigm. Annu Rev Resour Econ. 2009;1(1):15-31.

6. Redding S, Schott PK. Distance, skill deepening and development: will peripheral countries ever get rich? J Dev Econ. 2003;72(2):515-41.

7. Kremer M. The O-ring theory of economic development. Q J Econ. 1993;108(3):551-75.

8. ILO India Labour Market Update, ILO Country Office for India, International Labor Organization. July 2016, 2016.

9. von Grebmer K, Bernstein J, Nabarro D, Prasai N, Amin S, Yohannes Y, Sonntag A, Patterson F, Towey O. and J. Thompson. 2016 Global Hunger Index: Getting to Zero Hunger. Bonn: Welthungerhilfe, International Food Policy Research Institute, and Concern Worldwide; 2016.

10. Deaton A, Drèze J. Food and nutrition in India: facts and interpretations. Economic and Political Weekly, 2009, 42-65.

11. Patnaik U. The republic of hunger. Social Scientist, 2004, 9-35.

12. Patnaik U. Neoliberalism and rural poverty in India. Economic and Political Weekly, 2007, 31323150.

13. Sundaram K, Tendulkar SD. Poverty among social and economic groups in India in 1990s. Economic and Political Weekly, 2003, 5263-5276.

14. Nayak P, Oliveira L, Berkes F. Resource degradation, marginalization, and poverty in small-scale fisheries: threats to social-ecological resilience in India and Brazil. Ecology and Society, 19(2), 2014.

15. Béné $C$. When fishery rhymes with poverty: a first step beyond the old paradigm on poverty in smallscale fisheries. World Dev. 2003;31(6):949-75. 
16. Gillespie S, Harris J, Kadiyala S The Agriculture-Nutrition Disconnect in India: What Do We Know? Technical Report. IFPRI Discussion Paper, Washington DC, 2012.

17. Parthasarathy G. Recent trends in wages and employment of agricultural labour. Indian J Agric Econ. 1996;51(1):145.

18. Rajuladevi AK. Food poverty and consumption among landless labour households. Economic and Political Weekly, 2001, 2656-2664.

19. Irz X, Lin L, Thirtle C, Wiggins S. Agricultural productivity growth and poverty alleviation. Development Policy Review. 2001;19(4):449-66.

20. Cecchini S, Scott C. Can information and communications technology applications contribute to poverty reduction? Lessons from rural India. Information Technology for Development. 2003;10(2):73-84.

21. Dorward A, Kydd J, Morrison J, Urey I. A policy agenda for pro-poor agricultural growth. World Dev. 2004;32(1):73-89.

22. Ruel MT, Alderman H, Maternal. Child Nutrition Study Group. (2013). Nutrition-sensitive interventions and programmes: how can they help to accelerate progress in improving maternal and child nutrition? The Lancet, 382(9891), 536-51.

23. Prein $M$, Ahmed $M$. Integration of aquaculture into smallholder farming systems for improved food security and household nutrition. FoodNutr Bull. 2000;21(4):466-71.

24. Bryce J, Coitinho D, Darnton-Hill I, Pelletier D, Pinstrup-Andersen P. \& Maternal and Child Undernutrition Study Group. Maternal and child undernutrition: effective action at national level. The Lancet. 2008;371(9611):510-26.

25. Dreze J, Sen A. Hunger and public action. New Delhi: Oxford University Press; 1989.

26. Paul VK, et al. Reproductive health, and child health and nutrition in India: meeting the challenge. The Lancet. 2011;377(9762):332-49.

27. Diao X, Hazell P, Thurlow J. The role of agriculture in African development. World Dev. 2010;38(10):1375-83.

28. Garrity DP, et al. Evergreen Agriculture: a robust approach to sustainable food security in Africa. Food Security. 2010;2(3):197-214.

29. Zezza A, Tasciotti L. Urban agriculture, poverty, and food security: empirical evidence from a sample of developing countries. Food Policy. 2010;35(4):265-73.

30. NCO, National Classification of Occupations. 2004, Directorate General of Employment, Ministry of Labour \& Employment, Government of India, 2004.

31. NSSO, Nutritional Intake in India 2011-12, NSS 68th Round, National Sample Survey Office, Ministry of Statistics and Programme Implementation, Government of India, 2014.

32. IIPS \& Macro International. India National Family Health Survey (NFHS-3), 2005-06. Vol. 1. Mumbai: International Institute for Population Sciences; 2007. 
33. Browne WJ, Subramanian SV, Jones K, Goldstein H. Variance partitioning in multilevel logistic models that exhibit over dispersion. Journal of the Royal Statistical Society: Series A (Statistics in Society). 2005;168(3):599-613.

34. Goldstein H, Browne W, Rasbash J. Partitioning variation in multilevel models. Understanding Statistics: Statistical Issues in Psychology, Education, and the Social Sciences, 1(4), 2002, 223-231.

35. Stata Corp LP. Stata Statistical Software Release 9. Stata Press Publication, 2005.

36. Leckie G, Charlton C. Runmlwin-a program to run the MLwiN multilevel modeling software from within stata. J Stat Softw. 2013;52(11):1-40.

37. Charlton C, Rasbash J, Browne WJ, Healy M, Cameron B MLwiN Version 3.00. Centre for Multilevel Modeling, University of Bristol, 2017.

38. Kurien J. The Kerala model: its central tendency and the outlier. Social Scientist, 1995, 70-90.

39. Mehta AK, Shah A. Chronic poverty in India: Incidence, causes and policies. World Dev. 2003;31(3):491-511.

40. Dev SM. Inclusive Growth in India: Agriculture, poverty and human development. New Delhi: Oxford University Press; 2010.

41. Dubey A, Gangopadhyay S, Wadhwa W. Occupational structure and incidence of poverty in Indian towns of different sizes. Rev Dev Econ. 2001;5(1):49-59.

42. Datt $\mathrm{G}$, Ravallion $\mathrm{M}$. Why have some Indian states done better than others at reducing rural poverty? Economica. 1998;65(257):17-38.

43. Datt G. (1998). Poverty in India and Indian states: an update. The Indian Journal of Labour Economics, 41(2), 41, 1998.

44. Ravallion M, Datt G. Why has economic growth been more pro-poor in some states of India than others? J Dev Econ. 2002;68(2):381-400.

45. Dev SM, Ravi C. Poverty and inequality: All-India and states, 1983-2005. Economic and Political Weekly, 2007, 509-521.

46. Bhanumurthy NR, Mitra A. Economic growth, poverty and inequality in Indian states in the pre-reform and reform periods. Asian Dev Rev. 2004;21(2):79.

47. Meenakshi JV, Vishwanathan B. Calorie Deprivation in Rural India, 1983-1999/2000. Economic and Political Weekly, 2003, 369-375.

48. United Nations, Department of Economic and Social Affairs. Population Division (World Population Prospects: The 2015 Revision, Methodology of the United Nations Population Estimates and Projections. ESA/P/WP.242, 2015.

49. RGI. Census of India. 2011. Provisional Population Totals. The Registrar General of India, New Delhi: Government of India, 2011.

50. Bloom DE, Williamson JG. Demographic Transitions and Economic Miracles in Emerging Asia. World Bank Economic Review. 1998;12(3):419-56. 
51. Bloom DE, Canning D, Sevilla J. The Demographic Dividend: A New Perspective on the Economic Consequences of Population Change, Population Matters Monograph MR-1274. Santa Monica: RAND; 2003.

52. Mason A, Lee R. Reform and support systems for the elderly in developing countries: Capturing the second demographic dividend. Genus LXII. 2006;(2):11-35.

53. James KS, Glorifying, Malthus. Current debate on 'demographic dividend' in India. Economic Political Weekly. 2008;June 21:63-9.

54. Chandrasekhar CPJ, Ghosh. and A. Roy Chowdhury The Demographic Dividend and Young India's Economic Future. Economic Political Weekly. 2006;December 9(9):5055-64.

55. Bloom DE Population dynamics in India and implications for economic growth, PGDA Working Paper 65, Harvard School of Public Health, Harvard University, Boston, MA. 2011.

56. Thomas JJ. The demographic challenge and employment growth in India. Economic Political Weekly. 2014;49(6):15-7.

57. Oommen MA, Micro Finance, Alleviation P: The Case of Kerala's Kudumbashree. Centre for SocioEconomic \& Environmental Studies, 2008.

58. Williams G, Thampi BV, Narayana D, Nandigama S, Bhattacharyya D. Performing participatory citizenship-politics and power in Kerala's Kudumbashree programme. Journal of Development Studies. 2011;47(8):1261-80.

59. Béné $C$, Hersoug B, Allison EH. Not by rent alone: analyzing the pro-poor functions of small scale fisheries in developing countries. Development Policy Review. 2010;28(3):325-58.

60. Béné C, Friend RM. Poverty in small-scale fisheries: old issue, new analysis. Progress in Development Studies. 2011;11(2):119-44.

61. Joshi PK, Gulat A, Birthal PS, Tewari L. Agriculture diversification in South Asia: patterns, determinants and policy implications. Economic and Political Weekly, 2004, 2457-2467.

62. Roemer M. Fishing for growth: export-led development in Peru, 1950-1967. Harvard University Press, 1970.

63. Thorpe A, Ibarra AA, Reid C. The new economic model and marine fisheries development in Latin America. World Dev. 2000;28(9):1689-702.

64. Ibarra AA, Reid C, Thorpe A. The political economy of marine fisheries development in Peru, Chile and Mexico. Journal of Latin American Studies. 2000;32(2):503-27.

65. Golub S, Varma A. Fishing exports and economic development of least developed countries: Bangladesh, Cambodia, Comoros, Sierra Leone and Uganda. UNCTAD. Swarthmore College, 2014.

66. Smith LC. The great Indian calorie debate: Explaining rising undernourishment during India's rapid economic growth. Food Policy. 2015;50:53-67.

\section{Figures}




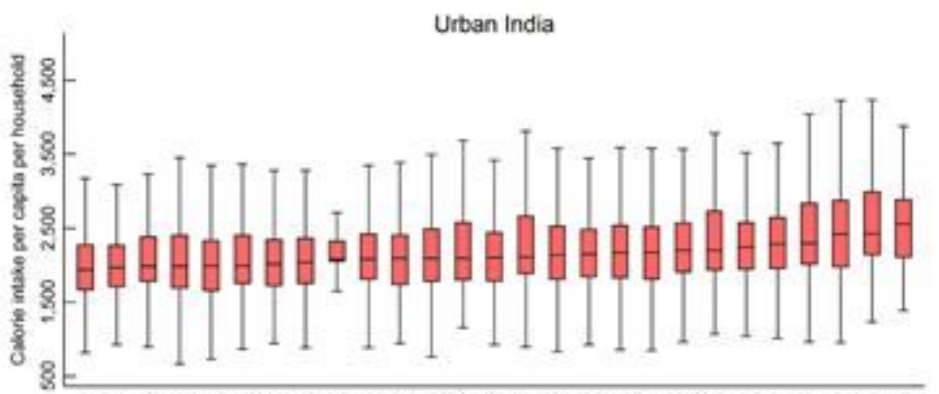

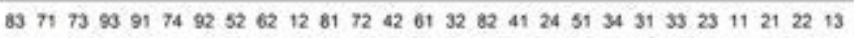
exiludes outside values

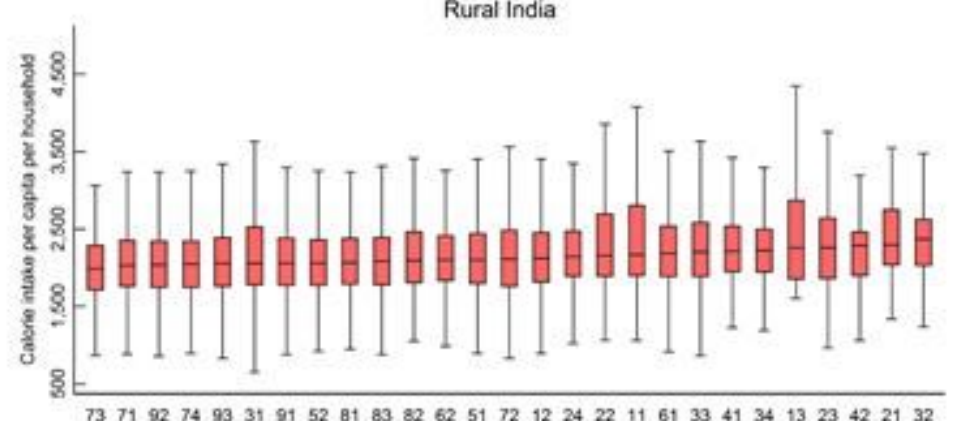

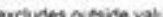

- NCO 2-digit codes: 11 Legislators and Senios Officiak, 12 Corporate Maragers, 13 General Manazers, 21 Scieace Professionak, 22 Life Science and Health Professionak, 23 Teaching Professionak, 24 Other Professionak. 31 Science Associase Professionak, 32 Life Science and Health Associale Professionak, 33 Teaching Associuse Professionak, 34 Other Asockiste Professionak, 41 Office Clerks, 42 Cestomer Senvices Clerks, si Persomal and Probective Service Workers, 52 Modek, Sales Persons and Demonotrasors, 61 Market Oriented Skilled Agricultural and Fichery Workers, 62 Suboistence Asricultural and Fichery Workers, 71 Fstraction and Brilding Frades Workers, 72 Meial, Machinery and Relaked Trades Workers. 73 Precision. Handicraft, Printing and Relased Irades Workers, 74 Other Craft and Relased Trades Workers, 81 Stationary

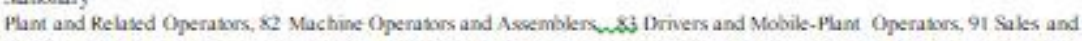
Services Elementary Occupations. 92 Agricultural. Fibhery and Relased Labourers, 93 Mining. Constraction. Manufacturing and Transport Labourers

Nole: Outliers omithed for comemience of exposition.

\section{Figure 1}

Box-plots, calorie intake per capita per household by NCO occupational classification in rural and urban India, National Sample Survey, 2011-2012. 

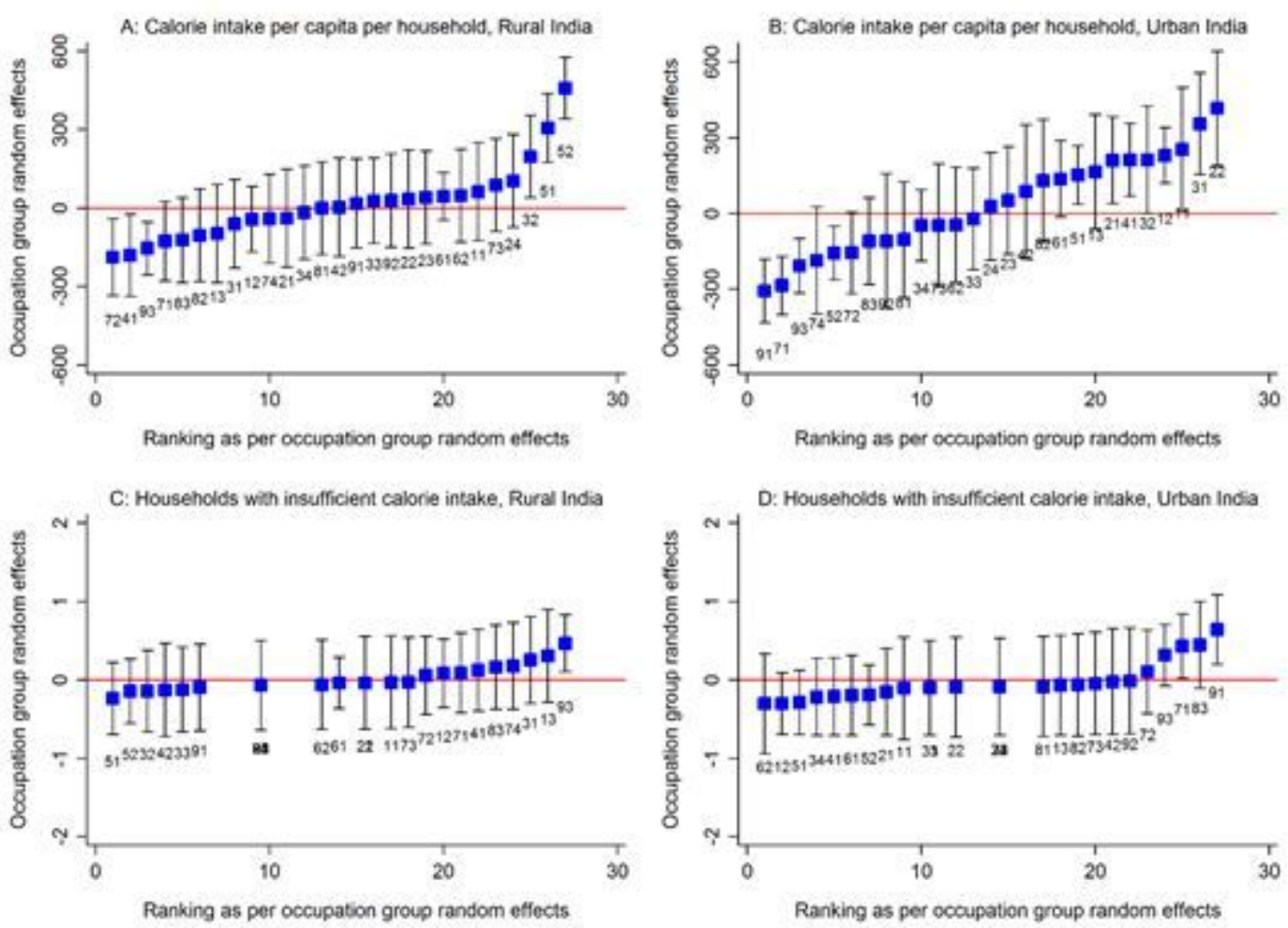

Note: The occupation group random-effects are bas ed onnull models with sampling weights and standarderrors clustered at the NCO occupational group, district, region and state level.

NCO 2-digit codes: 11 Legislators and Senior Officials, 12 Corporate Managers, 13 General Managers, 21 Science Professionals, $22 \mathrm{~L}$ ife Science and Health Professionals, 23 Teaching Professionals, 24 Other Professionals, 31 Science As sociate Professionals, $32 \mathrm{~L}$ ife Science and Health As sociate Professionals, 33 Teaching Ass ociate Professionals, 34 Other As sociate Professionals, 41 Office Clerks, 42 Customer Services Clerks, 51 Pers onal and Protective Service Workers, 52 Models, Sales Pers ons and Demonstrators, 61 Market Oriented SkilledAgricultural and Fishery Workers, 62 Subsistence Agricultural and Fishery Workers, 71 Extraction andBuilding Trades Workers, 72 Metal, Machinery and Related Trades Workers, 73 Precision, Handicraft, Printing and Related Trades Workers, 74 Other Craft and Related Trades Workers, 81 Stationary Plant and Related Operators, 82 Machine Operators and Assemblers, 83 Drivers and Mobile-Plant Operators, 91 Sales and Services Elementary Occupations, 92 Agricultural, Fishery and Related Labourers, 93 Mining, Construction, Manufacturing and Transport Labourers

\section{Figure 2}

Caterpillar plot of random-intercept predictions $(95 \% \mathrm{Cl})$ versus ranking of occupation group effects for average calorie intake per capita per household and undernourished households for rural and urban India, (National Sample Survey, India 2011-12) 